\title{
Evaluation of Software Fault Prediction Models Considering Faultless Cases*
}

\author{
Yukasa MURAKAMI ${ }^{\dagger}$, Nonmember, Masateru TSUNODA $^{\dagger a}{ }^{\text {, }}$, and Koji TODA $^{\dagger \dagger}$, Members
}

\begin{abstract}
SUMMARY To enhance the prediction accuracy of the number of faults, many studies proposed various prediction models. The model is built using a dataset collected in past projects, and the number of faults is predicted using the model and the data of the current project. Datasets sometimes have many data points where the dependent variable, i.e., the number of faults is zero. When a multiple linear regression model is made using the dataset, the model may not be built properly. To avoid the problem, the Tobit model is considered to be effective when predicting software faults. The model assumes that the range of a dependent variable is limited and the model is built based on the assumption. Similar to the Tobit model, the Poisson regression model assumes there are many data points whose value is zero on the dependent variable. Also, log-transformation is sometimes applied to enhance the accuracy of the model. Additionally, ensemble methods are effective to enhance prediction accuracy of the models. We evaluated the prediction accuracy of the methods separately, when the number of faults is zero and not zero. In the experiment, our proposed ensemble method showed the highest accuracy, and Pred25 was $21 \%$ when the number of faults was not zero, and it was $45 \%$ when the number was zero.

key words: defect prediction, Tobit model, Poisson regression, ensemble method
\end{abstract}

\section{Introduction}

In a large scale software development project, management is important, to avoid failure of it. In project management, planning is based on the prediction of the project. For example, the software testing plan is made based on software fault prediction. Therefore, the accuracy of the prediction is important. To enhance the prediction accuracy of the number of faults, various models are proposed [11], [13]. The model is built based on a dataset of past projects, and it is predicted using the model and the data of the current project. For instance, the programming language and software size of a current project are input to the model, and using them, the number of faults is predicted. To make the prediction model, multiple linear regression model is widely used.

However, some datasets have many data points where

\footnotetext{
Manuscript received August 19, 2019.

Manuscript revised January 27, 2020.

Manuscript publicized March 9, 2020.

${ }^{\dagger}$ The authors are with Kindai University, Higashiosaka-shi, 577-8502 Japan.

${ }^{\dagger \dagger}$ The author is with Fukuoka Institute of Technology University, Fukuoka-shi, 811-0295 Japan.

*This work is an extended study of M. Tsunoda et al., "An empirical evaluation of the Tobit model on software defect prediction?" Proc. of Applied Computing and Information Technology (ACIT), pp.196-201, 2016.

a)E-mail: tsunoda@info.kindai.ac.jp

DOI: 10.1587/transinf.2019KBP0019
}

the number of faults is zero and a major independent variable such as software size is not zero. In this case, when a multiple linear regression model is built based on ordinary least squares, it may not be built appropriately. For example, a model is built using the dataset in which the number of faults is zero (i.e., faultless) when software size is smaller than 100 FP (Function Point). Then, the model may predict that the number of faults is smaller than zero, when software size of a current project is much smaller than $100 \mathrm{FP}$.

To avoid the problem, the Tobit model [31] is considered to be effective when predicting software faults. It is widely used in other fields such as quantitative sociology. It assumes that the range of a dependent variable is limited, e.g., the minimum value of the variable is zero, and the model is built based on the assumption. Although the built model is similar to the linear regression model, the Tobit model uses another model when the dependent variable is smaller than the lower bound of it. The assumption seems well fit to software fault prediction. So, applying the Tobit model is expected to enhance prediction accuracy of software fault prediction. Although the Tobit model was not very new method, the performance of the model has not been evaluated on software fault prediction, as far as we know.

There are other prediction methods to predict software faults. Similar to the Tobit model, the Poisson regression model assumes there are many data points whose value is zero on the dependent variable. The Poisson regression model is sometimes used to build a fault prediction model [12]. Also, when building a model which predicts software development effort or the number of faults, log-transformation is sometimes applied, to enhance the accuracy of the model [15]. This is because the distributions of some variables are log-normal distribution. Additionally, combining multiple prediction models (i.e., ensemble methods) is effective to enhance prediction accuracy of the models [17].

To the best of our knowledge, the performances of prediction methods have not been empirically evaluated (e.g., through a comparison between the Tobit model and the Poisson regression model), and therefore, it is not clear that which method is effective to predict software faults. So, we use a dataset collected from actual software development companies, and evaluate the prediction accuracy of the methods. In the experiment, the number of faults found after the release of software is predicted. The number of faults are often zero, i.e., there are many cases whose fault is zero 
(faultless cases). This means when a models predicts that the number of faults of all cases is zero, the performance of the model seems high erroneously. So, we evaluate the prediction methods separately, when the number of faults is zero and not zero.

In this paper, two major contributions are described. First, we newly applied the Tobit model to a software fault prediction, and compared the performance with other models such as a Poisson regression model, when considering faultless cases. Second, a new ensemble method that combines the Tobit model with other models is proposed.

In Sect. 2, existing and proposed fault prediction methods are described. Section 3 reports an experiment conducted to evaluate the accuracy of the prediction methods. Section 4 analyzes and discusses the results of the experiment. Section 5 introduces previous related studies. Finally, Sect. 6 provides some concluding remarks regarding this study.

\section{Prediction Methods}

In Sects. 2.1, 2.2, and 2.4, we describe the existing software fault prediction methods, and in Sects. 2.3 and 2.5 we introduce the newly proposed fault prediction methods.

The models predict the quality of the entire software product [2], [5], and are therefore useful to make a project plan and manage projects. Concretely speaking, based on the prediction results, we can determine the organization of a software development project and the maintenance phase [5]. For example, when a model predicts that numerous faults will be found in a particular type of software, a project manager can assign a larger maintenance staff after the software release than normally applied prior to its release.

\subsection{Multiple Linear Regression Model}

The multiple linear regression model is widely used when predicting the number of software faults mathematically. The model is built based on ordinary least squares. When the number of faults is denoted as $y$, and independent variables such as software size are denoted as $x_{1}, x_{2}, \ldots, x_{k}(k$ is the number of independent variables), $y$ is explained as:

$$
y=\beta_{0}+\beta_{1} x_{1}+\beta_{2} x_{2}+\ldots+\beta_{k} x_{k}+\varepsilon
$$

In the equation, $\beta_{0}$ is an intercept, $\beta_{1}, \beta_{2}, \ldots, \beta_{k}$ are partial regression coefficients, and $\varepsilon$ is an error term. As a rule of thumb, to build a proper model using linear regression analysis, it is needed that the number of data points is five to ten times larger than the number of independent variables.

\subsection{Log-Transformation}

When building a model which predicts software development effort or the number of faults, log-transformation is sometimes applied, to enhance the accuracy of the model [15]. This is because the distributions of some variables are log-normal distribution. Predicted values are larger than zero, when log-transformation is applied to the dependent variable. That is, the predicted value of development effort and the number of faults is larger than zero. So, the model predicts that the number of faults is larger than zero, even when software size of a current project is very small.

\subsection{Tobit Model}

The Tobit model [31] focuses the bias of the distribution of the dependent variable, when building a model. The Tobit model classifies the bias into the followings [24].

- Censored

- Truncated

- Incidental truncation

Censored means the range of the dependent variable is limited (e.g., the minimum value of the variable is zero). For instance, the number of software faults is censored data. Truncated means some data points (e.g., data points whose number of faults are zero) are excluded from the dataset for some reason. Incidental truncation means the values of some data points are zero, although the original values are not zero. For example, the number of found faults in the code review is zero when the review is skipped. When data is regarded as censored or truncated, the type I Tobit model is applied, and when it is regarded as incidental truncation, the type II Tobit model is applied. To predict the number of faults, the type I Tobit model is applied.

Type I Tobit model includes the censored regression model and the truncated regression model. The censored regression model is denoted as:

$$
\begin{aligned}
& y^{*}=\beta_{0}+\beta_{1} x_{1}+\beta_{2} x_{2}+\ldots+\beta_{k} x_{k}+\varepsilon \\
& y= \begin{cases}y^{*}, & y^{*}>0 \\
0, & y^{*} \leq 0\end{cases}
\end{aligned}
$$

In the equation, $\beta_{0}$ is an intercept, $\beta_{1}, \beta_{2}, \ldots, \beta_{k}$ are partial regression coefficients, and $\varepsilon$ is an error term. We use the censored regression model to predict software faults. Although the Tobit model was not very new method, the performance has not been evaluated on software fault prediction, as far as we know.

Figure 1 illustrates the Tobit model and the linear

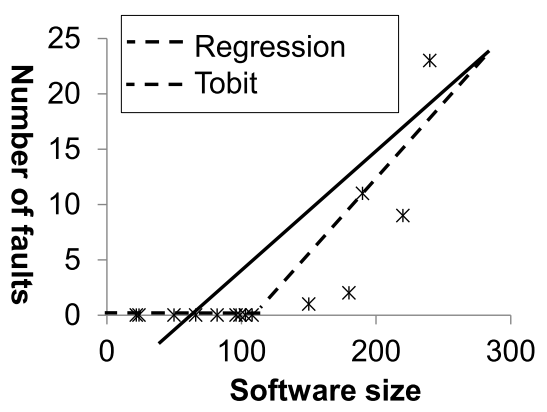

Fig. 1 The Tobit model and the regression model 
regression model based on ordinary least squares. In the figure, the independent variable ( $\mathrm{x}$-axis) is software size, and the dependent variable ( $\mathrm{y}$-axis) is the number of software faults. Points in the graph are data points of projects. As illustrated in the figure, the Tobit model properly treats data points whose number of faults are zero.

As shown in Eqs. (2) and (3), the Tobit model assumes that the relationships between independent variables and a dependent variable does not change, regardless of whether the predicted value $y^{*}$ is larger than zero. When we use the independent variables shown in Table 2, the relationships are not considered to have changed, regardless of whether the value of $y^{*}$ is larger than zero. For instance, as described in Sect.3.2, the number of faults and the software size (FP) are positively correlated (correlation coefficient of 0.43 ), when the number of faults is larger than zero. The relationship will not change (i.e., the faults and their size will not become negatively correlated), when the number of faults is zero (i.e., faultless cases). This is because there are no special reasons for the number of faults to reach zero during the software development. As the simple reason for this number, the software is small in size. Therefore, applying the Tobit model to a software fault prediction is valid.

\subsection{Poisson Regression}

Similar to the Tobit model, the Poisson regression model assumes there are many data points whose value is zero on the dependent variable. The Poisson regression model is sometimes used to build a fault prediction model [12]. In more detail, the Poisson regression model assumes the distribution of the dependent variable is the Poisson distribution. On the Poisson distribution, the average and the variance is same. For example, the number of calls on a call center per hour is the Poisson distribution. When the number of faults is denoted as $y$, and independent variables are denoted as $x_{1}, x_{2}, \ldots, x_{k}, y$ is explained as:

$$
\ln (y)=\beta_{0}+\beta_{1} x_{1}+\beta_{2} x_{2}+\ldots+\beta_{k} x_{k}+\varepsilon
$$

In the equation, $\ln (y)$ is $\log$-transformed $y$. We can apply a Poisson regression appropriately when the average value of the dependent variable and its variance are almost same (i.e., a Poisson distribution). Also, the dependent variable should be integer, since the distribution is the Poisson distribution.

\subsection{Ensemble Methods}

Ensemble methods combine multiple prediction models using some algorithms. It is effective to enhance prediction accuracy of the models [17]. The simplest method calculates the average of predicted values derived from multiple prediction models, and uses it as the predicted value. In this paper, we call this simple ensemble. Based on the analysis described in Sect. 4.2, we combines the Tobit model and Poisson regression, or the Tobit model and the multiple linear regression model.
Table 1 Examples of ensemble methods

\begin{tabular}{l|l|l}
\hline & $\begin{array}{l}\text { Predicted value } \\
\text { of case A }\end{array}$ & $\begin{array}{l}\text { Predicted value } \\
\text { of case B }\end{array}$ \\
\hline Tobit model & 0 & 3 \\
Regression model & 5 & 7 \\
Simple ensemble & $(5+0) / 2=2.5$ & $(3+7) / 2=5$ \\
Selection ensemble & 0 & 7 \\
Average ensemble & $(5+0) / 2=2.5$ & 7 \\
\hline
\end{tabular}

To combine two prediction models, we propose two methods, based on the analysis described in Sect. 4.2. One of the combination models makes prediction as follows:

1. Select predicted value of the multiple linear regression model (or Poisson regression), when predicted value of the Tobit model is not zero.

2. Select predicted value of the Tobit model, when predicted value of the Tobit modes is zero.

The other combination models makes prediction as follows:

1. Select predicted value of the multiple linear regression model (or Poisson regression), when predicted value of the Tobit model is not zero.

2. Select the average of predicted values of the multiple linear regression model (or Poisson regression) and the Tobit model, when predicted value of the Tobit modes is zero.

In this paper, we call the former method selection ensemble, and call the latter method average ensemble. Table 1 shows examples of the ensemble methods when case A and B are predicted. As explained in Sect. 4.2, the prediction accuracy of the Tobit model is high, when the number of faults is zero. In contrast, the prediction accuracy of the multiple linear regression model and Poisson regression is high, when the number of faults is not zero. So, we combined the methods, focusing on faultless cases.

\section{Experiment}

\subsection{Overview}

To evaluate performance of the prediction models, we made prediction models using a dataset collected from actual software development companies. The number of faults found after the release of software was predicted using the models, and we evaluated the prediction accuracy of the models. We built the following prediction models:

- Multiple linear regression model (Linear regression)

- Tobit model

- Poisson regression

Also, we built the multiple linear regression model and the Tobit model, applying log-transformation to both independent variables and dependent variable. We added one to the dependent variable before the log-transformed. 
This is because the number of faults includes zero, and logtransformation cannot be applied to it. Adding one is common way to avoid the problem. Note that we did not apply the transformation to the dependent variable, when the Poisson regression model was built. Since it is logtransformed in the model, as explained in Sect. 2.4.

As a result, we built six types of the prediction models in the experiment. After analyzing prediction performance of the six models, we built three types of the ensemble prediction models, i.e., simple ensemble, selection ensemble, and average ensemble, and evaluated the prediction accuracy of them.

\subsection{Dataset}

To build the prediction models, we used the dataset provided by ISBSG (International Software Benchmarking Standards Group) [9]. Included projects were collected from software development companies in 20 countries. It is widely used to evaluate prediction models [22]. Version of the dataset is Release 9, and it includes the projects which were carried out between 1989 and 2004.

The dataset has 3026 projects and 99 variables, and there are many missing values. To uniform the data points used to build models, we selected projects whose data quality is A or B, and FP measurement method is IFPUG (International Function Point Users Group) method. The selection criteria is often applied by many studies [21]. Additionally, we eliminated data points which have missing values (i.e., listwise deletion was applied). As a result, 221 data points were selected, and the number of data points whose number of faults was zero was 86 . Figure 2 shows the distributions of the number of faults.

Figure 3 shows the relationship between FP and the number of faults. The Spearman rank correlation between them was 0.38 , and when we removed the data points whose fault was zero, the correlation was 0.43 . This result suggests that it is not easy to predict the number of faults based on FP.

The dependent variable is the number of faults, and candidates of independent variables are shown in Table 2 (except for the number of faults). We use variables such as platform and business sector, since we do not predict faultprone module but predict fault-prone project. In the dataset, code metrics are not recorded. However, business sector relates to the quality of the software to some extent. For example, if the business sector is banking, the quality of the software will be high. So, variables such as business sector are useful to predict faults.

The software size (i.e., the FP) is widely used as an independent variable in studies estimating the effort required for software development. González-Ladrón-de-Guevara et al. [6] showed that $61.7 \%$ of the studies using the ISBSG dataset treated the software size as an independent variable. Such studies assumed that the FP is settled after a basic design phase. Therefore, our study can be applied after this phase.

Nominal scale variables were transformed into dummy

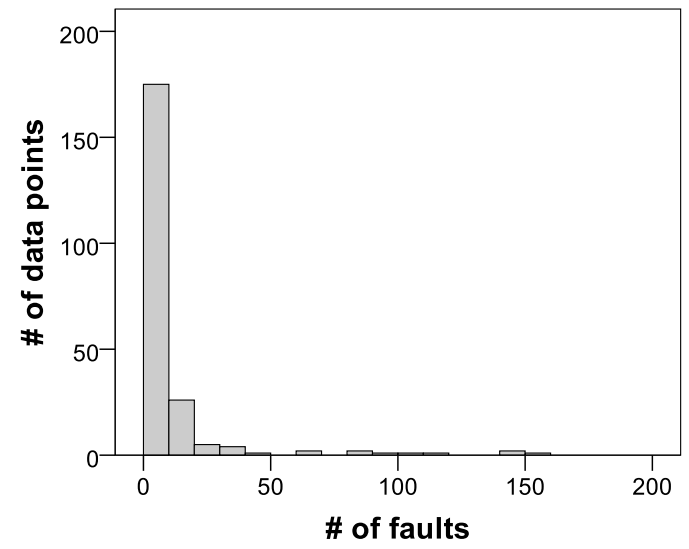

Fig. 2 The distribution of the number of the faults

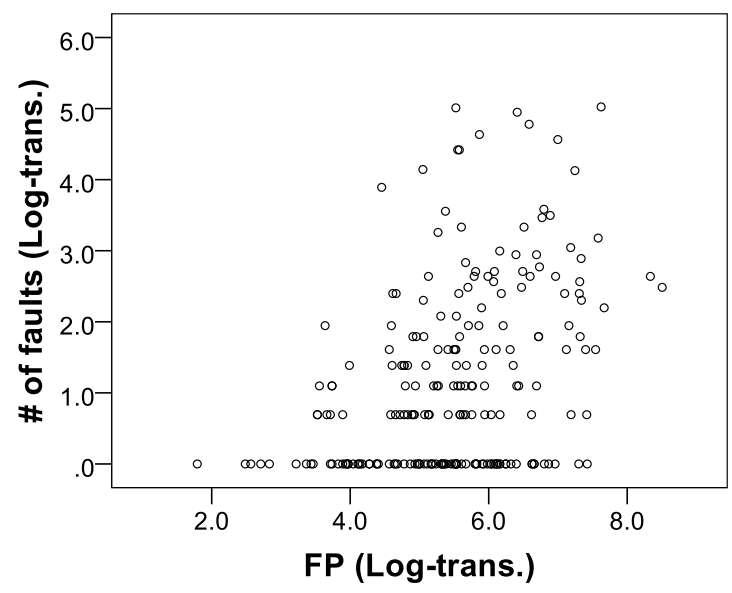

Fig. 3 The relationship between FP and the number of faults

Table 2 Variables used in the experiment

\begin{tabular}{|c|c|c|}
\hline Variable & Scale & Detail \\
\hline Number of faults & ratio & $\begin{array}{l}\text { Faults found after software } \\
\text { release within one month }\end{array}$ \\
\hline FP & ratio & Raw function points \\
\hline Development type & nominal & $\begin{array}{l}\text { Enhancement, and new } \\
\text { development }\end{array}$ \\
\hline Business area & nominal & $\begin{array}{l}\text { Banking, financial, insurance, } \\
\text { manufacturing, and others }\end{array}$ \\
\hline Platform & nominal & Mainframe, and midrange \\
\hline
\end{tabular}

variables (The value of the variable is 0 or 1 ). As a preliminary analysis, we performed variable selection using AIC (Akaike's Information Criterion) on the linear regression model based on ordinary least squares. Based on the result, we selected FP, new development (development type), banking (business sector), mainframe (platform), and midrange (platform) as the independent variables.

\subsection{Evaluation Criteria}

To evaluate prediction accuracy of the models, we used Pred25 [4], and average and median of $A E$ (Absolute Error), and $B R E$ (Balanced Relative Error) [23]. Note that in this paper, Pred25 indicates the ratio of data points whoso $B R E$ 
is smaller than $25 \%$. When $A E$ and $B R E$ is low and Pred 25 is high, prediction accuracy is regarded as high.

When $x$ denotes actual fault, and $\hat{x}$ denotes estimated fault, each criterion is calculated by the following equations. When the denominator of $B R E$ was zero, we added one to it.

$$
\begin{aligned}
& A E=|x-\hat{x}| \\
& B R E=\left\{\begin{array}{l}
\frac{|x-\hat{x}|}{|x|}, x-\hat{x} \geq 0 \\
\frac{|x-\hat{x}|}{|\hat{x}|}, x-\hat{x}<0
\end{array}\right.
\end{aligned}
$$

MRE (Magnitude of Relative Error) [4] is widely used to evaluate a prediction model, and MER (Magnitude of Error Relative to the estimate) [19] is sometimes used. However, MRE and MER are imbalanced for underestimation and overestimation [3], [20]. The maximum MRE is 1 even if an extreme underestimate occurs (For instance, when the actual fault is 1000 person-hour, and the estimated fault is 0 person-hour, MRE is 1). Similarly, maximum MER is smaller than 1 when an overestimate occurs. So, instead of $M R E$, we adopted $B R E$ whose evaluation is not biased [25]. The $B R E$ is often shown in percentile (e.g., in [32]), and therefore, we also show the $B R E$ in percentile, multiplying the values derived through Eq. (6) by 100 .

In the analysis, we focus on the Pred25. This is because $B R E$ is not very proper when the number of faults is zero, and $A E$ is also not very proper when the number of faults is not zero. In contrast, Pred25 is appropriate for evaluating both cases.

To build the models and calculate the evaluation criteria, we applied 5-fold cross validation. As shown in Fig. 2, the number of faults was zero (i.e., faultless cases) on many cases. So, when a models predicts that the number of faults of all cases is zero, the performance of the model seems high erroneously. To avoid the problem, we evaluated the predic- tion accuracy of the models separately, when the number of faults is zero and not zero. For the reference, we showed the accuracy of the model which always predicts the number of faults is zero.

\section{Results}

\subsection{Prediction Accuracy without Considering Faultless Cases}

To analyze the influence of the faultless cases (i.e., the number of faults was zero), we show prediction accuracy of the models when the results were not separated based on the number of faults. Table 3 shows the accuracy of the models when log-transformation is not applied. As described in Sect. 3.3, the average and median BRE are shown in percentile. In the table, the model which always predicts the number of faults is zero (All zero) seems high accuracy, if we do not care for average BRE. Table 4 shows the prediction accuracy separated based on the number of faults. In contrast, the accuracy of the model (All zero) is very low, when the number of faults is not zero. The results suggests that we should avoid evaluating the fault prediction models without considering faultless cases.

\subsection{Comparison of the Prediction Models}

Table 4 shows the prediction accuracy separated based on the number of faults. First, we evaluated effectiveness of log-transformation (i.e., case (a)-(h)). Focusing on the linear regression and the Poisson regression, the prediction accuracy was high when log-transformation was applied (e.g., comparison of case (c) and (j)). Note that the accuracy of the Poisson regression was not improved very much by the logtransformation, when the number of faults was zero (i.e., between case (h) and (n)). For example, average $A E$ was

Table 3 Prediction accuracy of the models without considering faultless cases

\begin{tabular}{lrrrrr}
\hline & Average $\boldsymbol{A} \boldsymbol{E}$ & Median $\boldsymbol{A} \boldsymbol{E}$ & Average $\boldsymbol{B R} \boldsymbol{E}$ & Median $\boldsymbol{B R} \boldsymbol{E}$ & Pred25 \\
\hline Tobit model & 9.66 & 2.04 & $378 \%$ & $115 \%$ & $37 \%$ \\
Linear regression & 10.74 & 4.11 & $350 \%$ & $169 \%$ & $9 \%$ \\
Poisson regression & 12.83 & 4.00 & $366 \%$ & $285 \%$ & $7 \%$ \\
All zero & 8.84 & 1.30 & $884 \%$ & $130 \%$ & $39 \%$ \\
\hline
\end{tabular}

\begin{tabular}{|c|c|c|c|c|c|c|c|c|}
\hline Case & \# of faults & Log-trans. & Model & Average $A E$ & Median $A E$ & Average $B R E$ & Median $B R E$ & Pred25 \\
\hline (a) & $>0$ & $\mathrm{Y}$ & All Zero & 14.46 & 4.80 & $1446 \%$ & $480 \%$ & $0 \%$ \\
\hline (b) & $>0$ & $\mathrm{Y}$ & Tobit model & 13.15 & 3.93 & $358 \%$ & $171 \%$ & $10 \%$ \\
\hline (c) & $>0$ & Y & Linear regression & 12.31 & 3.40 & $254 \%$ & $101 \%$ & $26 \%$ \\
\hline (d) & $>0$ & Y & Poisson regression & 15.46 & 5.17 & $231 \%$ & $99 \%$ & $20 \%$ \\
\hline (e) & 0 & $\mathrm{Y}$ & All Zero & 0.00 & 0.00 & $0 \%$ & $0 \%$ & $100 \%$ \\
\hline (f) & 0 & $\mathrm{Y}$ & Tobit model & 1.03 & 0.14 & $103 \%$ & $14 \%$ & $56 \%$ \\
\hline (g) & 0 & $\mathrm{Y}$ & Linear regression & 1.79 & 1.18 & $179 \%$ & $118 \%$ & $13 \%$ \\
\hline (h) & 0 & $\mathrm{Y}$ & Poisson regression & 4.86 & 3.33 & $486 \%$ & $333 \%$ & $0 \%$ \\
\hline (i) & $>0$ & $\mathrm{~N}$ & Tobit model & 14.73 & 5.00 & $502 \%$ & $224 \%$ & $10 \%$ \\
\hline (j) & $>0$ & $\mathrm{~N}$ & Linear regression & 14.48 & 6.36 & $269 \%$ & $141 \%$ & $13 \%$ \\
\hline (k) & $>0$ & $\mathrm{~N}$ & Poisson regression & 17.70 & 5.56 & $277 \%$ & $114 \%$ & $12 \%$ \\
\hline (l) & 0 & $\mathrm{~N}$ & Tobit model & 1.59 & 0.00 & $159 \%$ & $0 \%$ & $80 \%$ \\
\hline (m) & 0 & $\mathrm{~N}$ & Linear regression & 4.68 & 2.42 & $468 \%$ & $242 \%$ & $2 \%$ \\
\hline (n) & 0 & $\mathrm{~N}$ & Poisson regression & 4.97 & 3.41 & $497 \%$ & $341 \%$ & $0 \%$ \\
\hline
\end{tabular}

Table 4 Prediction accuracy of the models separated by the number of faults 
Table 5 Prediction accuracy of the ensemble methods

\begin{tabular}{|c|c|c|c|c|c|c|c|c|}
\hline Case & \# of faults & Combined model & Ensemble method & Average $A E$ & Median $A E$ & Average $B R E$ & Median $B R E$ & Pred25 \\
\hline (A) & $>0$ & Linear regression & Simple & 12.70 & 3.61 & $290 \%$ & $123 \%$ & $17 \%$ \\
\hline (B) & 0 & Linear regression & Simple & 1.41 & 0.66 & $141 \%$ & $66 \%$ & $31 \%$ \\
\hline (C) & $>0$ & Linear regression & Selection & 12.42 & 3.46 & $278 \%$ & $113 \%$ & $21 \%$ \\
\hline (D) & 0 & Linear regression & Selection & 1.58 & 0.92 & $158 \%$ & $92 \%$ & $45 \%$ \\
\hline (E) & $>0$ & Linear regression & Average & 12.36 & 3.42 & $262 \%$ & $103 \%$ & $22 \%$ \\
\hline (F) & 0 & Linear regression & Average & 1.69 & 1.05 & $169 \%$ & $105 \%$ & $31 \%$ \\
\hline (G) & $>0$ & Poisson regression & Simple & 13.64 & 3.45 & $223 \%$ & $91 \%$ & $25 \%$ \\
\hline (H) & 0 & Poisson regression & Simple & 2.94 & 1.77 & $294 \%$ & $177 \%$ & $1 \%$ \\
\hline (I) & $>0$ & Poisson regression & Selection & 15.64 & 5.36 & $267 \%$ & $115 \%$ & $13 \%$ \\
\hline (J) & 0 & Poisson regression & Selection & 3.97 & 2.53 & $397 \%$ & $253 \%$ & $45 \%$ \\
\hline (K) & $>0$ & Poisson regression & Average & 15.49 & 5.31 & $234 \%$ & $99 \%$ & $19 \%$ \\
\hline (L) & 0 & Poisson regression & Average & 4.41 & 2.92 & $441 \%$ & $292 \%$ & $1 \%$ \\
\hline
\end{tabular}

improved from 4.97 to 4.86 .

Focusing on the Tobit model, the accuracy was improved very much by the log-transformation, when the number of faults was not zero (comparison of case (b) and (i)). For example, average BRE was improved from 502\% to $358 \%$. In contrast, the accuracy was not improved when the number of faults was zero (comparison of case (f) and (1)). For example, Pred25 was degraded from $80 \%$ to $56 \%$. Considering the balance of the accuracy between them, logtransformation is also effective on the Tobit model. So, we applied log-transformation when building the three ensemble prediction models.

When the number of faults was zero (and logtransformation was applied) (on case (f)-(h)), the accuracy of the Tobit model was the highest. For example, Pred25 of other models were lower than the Tobit model. In contrast, when the number of faults was not zero (on case (b)-(d)), the accuracy of the Tobit model was the lowest. So, we proposed the three ensemble prediction models explained in Sect. 2.5.

\subsection{Comparison of the Ensemble Models}

Table 5 shows the prediction accuracy of the ensemble methods, when combining the linear regression or the Poisson regression. When combining the Poisson regression and predicting faultless cases (i.e., the number of faults was zero; case (H), (J), and (L)), Pred25 was very low, and the prediction accuracy was not high. This might be because both the Tobit model and the Poisson regression model presume that a dependent variable includes data points whose values are zero, and the combinations of these models do not complement each other. In contrast, when combining the linear regression and predicting faultless cases (case (B), (D) and (F)), Pred25 was improved. That is, in Table 4, Pred25 was $13 \%$ (case $(\mathrm{g})$ ), and in Table 5, Pred25 was larger than $31 \%$. So, we focus on the ensemble methods using the linear regression (case $(\mathrm{A})-(\mathrm{F}))$.

When predicting the cases whose number of faults was not zero (case (A), (C), and (E)), Pred25 was $21 \%$ and Median BRE was the $113 \%$ on selection ensemble using the linear regression (case (C)), and it was not the lowest accuracy among the methods. Also, the method was not low accuracy when predicting faultless cases (case (D)). So, we

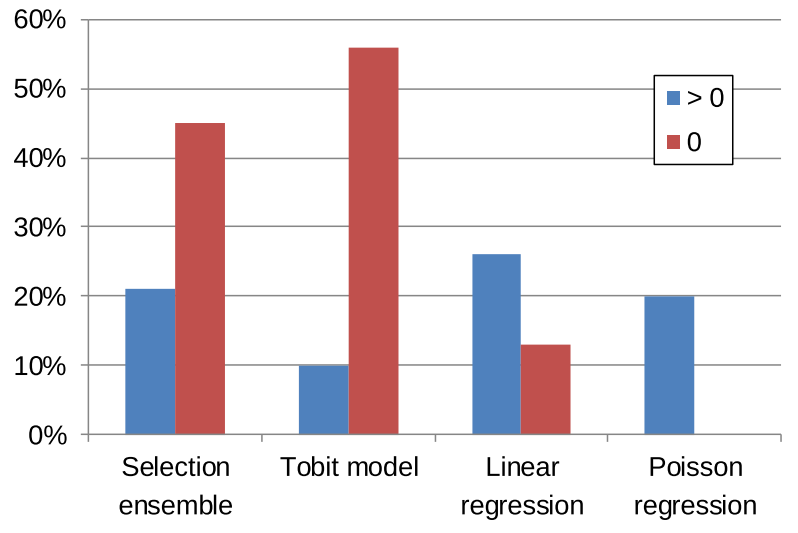

Fig. 4 Pred25 of prediction models with log-transformation

focus on the selection ensemble using the linear regression.

We compared the selection ensemble (case (C) and (D)) and other prediction models with log-transformation shown in Table 4 (case (b)-(d), and (f)-(h)). Pred25 of the selection ensemble was the second highest accuracy among them. That is, the prediction accuracy of the selection ensemble is stably higher when the number of faults is both zero and not zero. Figure 4 shows Pred 25 of the models.

\subsection{Overall Performance of Ensemble Models}

It is not clear whether a current project (i.e., prediction target) is actually faultless when using a prediction model. Therefore, the prediction models are not useful if the accuracy of the models of faultless cases (or other cases) is extremely low (e.g., Pred 25 is $0 \%$, and an example of such a case is described in Sect. 4.5). For this reason, we evaluated prediction models when considering faultless cases.

As a reference, we also show the ensemble models without a consideration of such cases in Table 6. All evaluation criteria of the selection ensemble in Table 6 are better than those of the linear regression model and the Poisson regression model in Table 3. When we compare the selection ensemble with the Tobit model in Table 3, the average $A E$, average $B R E$, and median $B R E$ are improved. In particular, the average $B R E$ was significantly improved. The difference in the median $A E$ is not large (a difference of 0.41). That is, the selection ensemble showed the best performance even 
Table 6 Prediction accuracy of the ensemble methods without considering faultless cases

\begin{tabular}{llrrrrr}
\hline Combined model & Ensemble method & Average $\boldsymbol{A} \boldsymbol{E}$ & Median $\boldsymbol{A} \boldsymbol{E}$ & Average $\boldsymbol{B R E}$ & Median $\boldsymbol{B R E}$ & Pred25 \\
\hline Linear regression & Simple & 8.37 & 2.41 & $235 \%$ & $99 \%$ & $22 \%$ \\
Linear regression & Selection & 8.27 & 2.45 & $234 \%$ & $106 \%$ & $30 \%$ \\
Linear regression & Ensemble & 8.28 & 2.46 & $228 \%$ & $103 \%$ & $25 \%$ \\
\hline Poisson regression & Simple & 9.47 & 2.73 & $252 \%$ & $124 \%$ & $16 \%$ \\
Poisson regression & Selection & 11.08 & 4.33 & $320 \%$ & $174 \%$ & $25 \%$ \\
Poisson regression & Ensemble & 11.15 & 4.41 & $316 \%$ & $176 \%$ & $12 \%$ \\
\hline
\end{tabular}

when we did not consider faultless cases.

\subsection{Discussion}

The results suggest that when we apply a selection ensemble using linear regression to software fault prediction, we should create a project plan and manage projects more properly based on the predicted number of faults, as compared with existing models. The prediction accuracy of the existing models is imbalanced. For example, the accuracy of the Poisson regression model is low when predicting faultless cases (i.e., Pred 25 is $0 \%$, as shown in Fig. 4). In contrast, the accuracy of the selection ensemble using linear regression was not low (i.e., Pred25 was 45\%, as shown in Fig. 4), even when predicting faultless cases. Hence, the selection ensemble is expected to suppress cases such as "We had assigned extra maintenance staff for post software release, based on predicted number of faults by the Poisson regression model. However, actual number of faults was zero (i.e., faultless), and the maintenance staff was excessive."

The limitation of the selection ensemble is the prediction accuracy for faulty cases (i.e., cases whose actual number of faults is zero). Compared with faultless cases, the prediction accuracy for faulty cases was not extremely high (i.e., Pred25 was $21 \%$, as shown in Fig. 4). Note that the accuracy is almost the same as that of the linear regression (Pred25 of the linear regression was 24\%). Therefore, we should make a project plan and manage projects when considering that the predicted number of faults includes errors to a certain extent, particularly when such number predicted by the selection ensemble is greater than zero.

\section{Related Work}

Many studies related to software fault prediction use models that discriminate whether each software module includes faults [1], [18]. Recently, some studies such as [26] have used a model predicting which methods will include faults (i.e., the granularity of the prediction is fine compared with the module-level prediction). Just-in-time fault prediction methods [10], [33] judge whether the source code includes faults when committed to a repository. Using the models described above, developers can identify the locations of the faults, which is useful for making a test plan and conducting debugging activities. In contrast, these models are unable to evaluate the quality of a software program. Similar to studies conducted by Bibi et al. [2] and Fenton et al. [5], our study focuses on the quality, and the prediction results are
Table 7 Average $A E$ of past study (regression via classification) [2]

\begin{tabular}{rrrrl}
\hline SMO & RIPPER & PART & C4.5 & IBk \\
\hline 9.08 & 9.77 & 9.56 & 9.36 & 10.17 \\
\hline
\end{tabular}

useful for making a project plan and managing a project [5].

As far as we know, the Tobit model was not applied to software fault prediction, and hence the performance of the model has not been evaluated. Shao et al. [28] used the Tobit model to analyze efficiency of investments about information technology. Sojer at al. [29] used the Tobit model to analyze developers' knowledge about internet code licenses. However, their research topics are different from software quality.

Some statistical models consider censored distribution of a variable. For example, survival analysis [16] considers censored data, and some papers [19], [27] used it to analyze characteristics of software such as the duration of open source project. However, the dependent variable in survival analysis is duration, and therefore, it is not used to predict the number of faults.

To predict software faults, some studies predicts that a module includes faults or not (i.e., applying discrimination models) [7]. This is because most of modules do not include faults, and some modules include few faults in the dataset used in the studies. The predicted results is mainly used to make a plan of software testing. In contrast, the range of number of faults is large in the dataset used in our study, as shown in Fig. 2. Therefore, applying discrimination models is not proper on our dataset. The predicted results of our study is mainly used to make a whole plan of a software development project.

Prediction accuracy of our models are not bad, compared with past studies (i.e., the difference in the average $A E$ is smaller than 0.6 between our model and previous studies). For example, Bibi et al. [2] predicted the number of faults using ISBSG dataset. Table 7 shows average $A E$ of their proposed method. They proposed a new method called regression via classification, and each column in the table shows combined model in their proposed method. Note that they did not show relative error such as BRE. Although selected projects were different from our experiment (They selected 97 projects), average $A E$ of Tobit model shown in Table 3 was almost same as Table 7 (i.e., that of the Tobit model is 9.66 and the minimum value shown in Table 7 is 9.08). Fenton et al. [5] predicted the number of residual faults using 31 projects collected from a consumer electronics company. In their experiment, Pred10 of their model was 26\%, and Pred30 was 58\%. Although we should aware that the 
dependent variable and dataset is different from our study, Pred 25 of Tobit model was not very bad, compared with the result.

\section{Conclusions}

In this paper, we evaluated software fault prediction models considering faultless cases. The number of faults are often zero in software project dataset. That is, there are many cases whose fault is zero (faultless cases). Therefore, when a models always predicts that the number of faults is zero, the performance of the model seems high erroneously. To avoid the problem, we evaluated the prediction models separately, when the number of faults is zero and not zero.

In the experiment, we evaluated the prediction accuracy of the multiple linear regression model, the Tobit model, and Poisson regression. As far as we know, the Tobit model was not applied to software fault prediction. Also, we evaluated the performance of log-transformation and ensemble methods. In this paper, we proposed two ensemble methods. The experimental results shows the followings:

- Log-transformation is effective to enhance prediction accuracy of models.

- The prediction accuracy of the linear regression and Poisson regression was high, when the number of faults is not zero.

- The prediction accuracy of the Tobit model was high, when the number of faults is zero.

- The prediction accuracy of an ensemble method which combines the linear regression and the Tobit model was well-balanced, when the number of faults is zero and not zero.

\section{Acknowledgments}

This research was partially supported by the Japan Society for the Promotion of Science (JSPS) [Grants-in-Aid for Scientific Research (A) (No.17H00731)].

\section{References}

[1] K.E. Bennin, J. Keung, P. Phannachitta, A. Monden, and S. Mensah, "MAHAKIL: Diversity Based Oversampling Approach to Alleviate the Class Imbalance Issue in Software Defect Prediction," IEEE Trans. Softw. Eng., vol.44, no.6, pp.534-550, 2018.

[2] S. Bibi, G. Tsoumakas, I. Stamelos, and I. Vlahavas, "Regression via Classification applied on software defect estimation," Expert Systems with Applications, vol.34, no.3, pp.2091-2101, 2008.

[3] C. Burgess and M. Lefley, "Can genetic programming improve software effort estimation? A comparative evaluation," Journal of Information and Software Technology, vol.43, no.14, pp.863-873, 2001.

[4] S. Conte, H. Dunsmore, and V. Shen, Software Engineering, Metrics and Models, Benjamin/Cummings, 1986.

[5] N. Fenton, M. Neil, W. Marsh, P. Hearty, Ł. Radliński, and P. Krause, "On the effectiveness of early life cycle defect prediction with Bayesian Nets," Empirical Software Engineering, vol.13, no.5, pp.499-537, 2008.

[6] F. González-Ladrón-de-Guevara, M. Fernández-Diego, and C.
Lokan, "The usage of ISBSG data fields in software effort estimation: A systematic mapping study," Journal of Systems and Software, vol.113, pp.188-215, 2016.

[7] T. Hall, S. Beecham, D. Bowes, D. Gray, and S. Counsell, "A Systematic Literature Review on Fault Prediction Performance in Software Engineering," IEEE Trans. Softw. Eng., vol.38, no.6, pp.1276-1304, 2012.

[8] H. Hata, O. Mizuno, and T. Kikuno, "Bug prediction based on finegrained module histories," Proc. International Conference on Software Engineering (ICSE), pp.200-210, 2012.

[9] International Software Benchmarking Standards Group, ISBSG Estimating, Benchmarking and Research Suite Release 9, ISBSG, 2004.

[10] Y. Kamei, E. Shihab, B. Adams, A.E. Hassan, A. Mockus, A. Sinha, and N. Ubayashi, "A Large-Scale Empirical Study of Just-InTime Quality Assurance," IEEE Trans. Softw. Eng., vol.39, no.6, pp.757-773, 2013.

[11] Y. Kastro and A. Bener, "A fault prediction method for software versioning," Software Quality Control, vol.16, no.4, pp.543-562, 2008.

[12] T. Khoshgoftaar, K. Gao, and R. Szabo, "An application of zero-inflated poisson regression for software fault prediction," Proc. International Symposium on Software Reliability Engineering (ISSRE), pp.66-73, 2001.

[13] T. Khoshgoftaar and N. Seliya, "Fault Prediction Modeling for Software Quality Estimation: Comparing Commonly Used Techniques," Empirical Software Engineering, vol.8, no.3, pp.255-283, 2003.

[14] B. Kitchenham, S. MacDonell, L. Pickard, and M. Shepperd, "What Accuracy Statistics Really Measure," Proc. IEE Software, vol.148, no.3, pp.81-85, 2001.

[15] B. Kitchenham and E. Mendes, "Why comparative effort prediction studies may be invalid," Proc. International Conference on Predictor Models in Software Engineering (PROMISE), art.4, p.5, 2009.

[16] J. Klein and M. Moeschberger, Survival Analysis: Techniques for Censored and Truncated Data, Springer, 2003.

[17] E. Kocaguneli, T. Menzies, and J. Keung, "On the value of ensemble effort estimation," IEEE Trans. Softw. Eng., vol.38, no.6, pp.1403-1416, 2012.

[18] M. Kondo, C. Bezemer, Y. Kamei, A. Hassan, and O. Mizuno, "The impact of feature reduction techniques on defect prediction models," Empirical Software Engineering, vol.24, no.4, pp.1925-1963, 2019.

[19] A. Koru, D. Zhang, and H. Liu, "Modeling the Effect of Size on Fault Proneness for Open-Source Software," Proc. International Workshop on Predictor Models in Software Engineering (PROMISE), p.10, 2007.

[20] C. Lokan, "What Should You Optimize When Building an Estimation Model?," Proc. International Software Metrics Symposium (METRICS), p.34, Como, Italy, Sept. 2005.

[21] C. Lokan and E. Mendes, "Cross-company and single-company effort models using the ISBSG Database: a further replicated study," Proc. International Symposium on Empirical Software Engineering (ISESE), pp.75-84, Rio de Janeiro, Brazil, Sept. 2006.

[22] E. Mendes, C. Lokan, R. Harrison, and C. Triggs, "A Replicated Comparison of Cross-company and Within-company Effort Estimation Models using the ISBSG Database," Proc. International Software Metrics Symposium (METRICS), p.36, 2005.

[23] Y. Miyazaki, M. Terakado, K. Ozaki, and H. Nozaki, "Robust Regression for Developing Software Estimation Models," Journal of Systems and Software, vol.27, no.1, pp.3-16, 1994.

[24] M. Mizuochi, "Introduction to Censored and Truncated Regression Models," Sociological Theory and Methods, vol.24, no.1, pp.129138, 2009 (in Japanese).

[25] K. Mølokken-Østvold and M. Jørgensen, "A Comparison of Software Project Overruns-Flexible versus Sequential Development Models,” IEEE Trans. Softw. Eng., vol.31, no.9, pp.754-766, 2005.

[26] L. Pascarella, F. Palomba, and A. Bacchelli, "Re-evaluating method-level bug prediction," Proc. International Conference on Software Analysis, Evolution and Reengineering (SANER), pp.592-601, 
2018.

[27] I. Samoladas, L. Angelis, and I. Stamelos, "Survival analysis on the duration of open source projects," Information and Software Technology, vol.52, no.9, pp.902-922, 2010.

[28] B. Shao and W. Lin, "Technical efficiency analysis of information technology investments: A two-stage empirical investigation," Information and Management, vol.39, no.5, pp.391-401, 2002.

[29] M. Sojer and J. Henkel, "License risks from ad hoc reuse of code from the internet," Communications of the ACM, vol.54, no.12, pp.74-81, 2011.

[30] M. Sokolova and G. Lapalme, "A systematic analysis of performance measures for classification tasks," Information Processing and Management, vol.45, no.4, pp.427-437, 2009.

[31] J. Tobin, "Estimation of relationships for limited dependent variables," Econometrica, vol.26, no.1, pp.24-36, 1956.

[32] M. Usman, E. Mendes, F. Weidt, and R. Britto, "Effort estimation in agile software development: a systematic literature review," Proc. International Conference on Predictive Models in Software Engineering (PROMISE), pp.82-91, 2014.

[33] X. Yang, D. Lo, X. Xia, Y. Zhang, and J. Sun, "Deep Learning for Just-in-Time Defect Prediction," Proc. International Conference on Software Quality, Reliability and Security, pp.17-26, 2015.

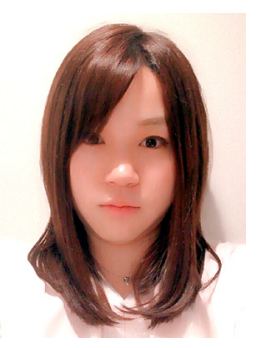

Yukasa Murakami received the BE (2016) and ME (2018) degrees in information science from Kindai University. Her research interests include human factors in software development, and project management.

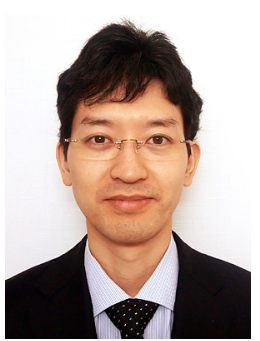

Masateru Tsunoda is an associate professor in Department of Informatics, Kindai University, Japan. He received the B.E. degree (1997) in economics from Wakayama University, and the M.E. (2004) and D.E. (2007) degrees in information science from Nara Institute of Science and Technology (NAIST). His research interests include software measurement, and human factors in software development. He is a member of IEICE, IEEE, IPSJ, JSSST, and JSiSE.

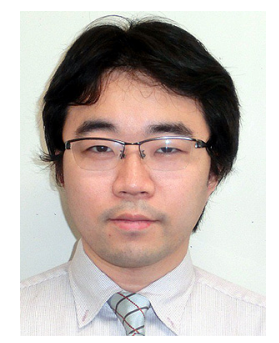

Koji Toda is an associate professor in Department of Computer Science and Engineering, Fukuoka Institute of Technology, Japan. He received the B.E. degree (2004) in engineering from Osaka University, and the M.E. (2006) and D.E. (2009) degrees in information science from Nara Institute of Science and Technology (NAIST). His research interests include software metrics. He is a member of IEICE, IEEE, and IPSJ. 